\title{
Editorial: The Magnetic Structures and Their Role in The Evolution of Coronal Mass Ejections
}

\author{
Hengqiang Feng ${ }^{1}$, Qiang $\mathrm{Hu}^{2 *}$ and Hongqiang Song ${ }^{3}$ \\ ${ }^{1}$ Institute of Space Physics, Luoyang Normal University, Luoyang, China, ${ }^{2}$ Department of Space Science and CSPAR, The \\ University of Alabama in Huntsville, Huntsville, AL, United States, ${ }^{3}$ Shandong Provincial Key Laboratory of Optical Astronomy and \\ Solar-Terrestrial Environment, and Institute of Space Sciences, Shandong University, Weihai, China
}

Keywords: heliophysics, space weather, coronal mass ejection, solar corona and wind, space physics, plasma physics

Editorial on the Research Topic

The Magnetic Structures and Their Role in The Evolution of Coronal Mass Ejections

Coronal mass ejections (CMEs) are the most energetic eruptions from the Sun and an important driver for space weather [1], which has significant impact on the technological systems of modern society. The aim of this research topic is to present multi-faceted research, from distinctive perspectives involving the forefront of Heliophysics-the science about the Sun, the Earth, and what's in-between, on the magnetic structures and other key ingredients of CMEs at different evolution stages. Multiple analysis tools, including theoretical, numerical, and observational ones, are employed, making use of a variety of ground-based and space-borne remote-sensing and in-situ measurements [2-5].

The magnetic structures of the solar source regions (usually the so-called active regions, i.e., ARs)

OPEN ACCESS

Edited and reviewed by:

Scott William McIntosh, National Center for Atmospheric Research (UCAR), United States

*Correspondence:

Qiang Hu

qiang.hu@uah.edu

Specialty section:

This article was submitted to Stellar and Solar Physics,

a section of the journal

Frontiers in Physics

Received: 23 November 2021 Accepted: 30 November 2021 Published: 21 December 2021

Citation:

Feng $H, H u Q$ and Song $H$ (2021) Editorial: The Magnetic Structures and Their Role in The Evolution of Coronal Mass Ejections.

Front. Phys. 9:820476. doi: 10.3389/fphy.2021.820476 are key to understanding the origination of CMEs [6-9]. Jiang et al. report a detailed analysis of the formation of a magnetic flux rope, the typical magnetic field configuration embodied by a CME, during the eruption process associated with solar flares/CMEs. They perform the state-of-the-art magnetohydrodynamic (MHD) numerical simulation for a general source region magnetic field topology. Their results reveal the corresponding topological change of the magnetic flux rope configuration. They find that the flux rope is formed via magnetic reconnection through a wellknown process, and additionally in the later phase, the reconnection proceeds between the field lines of the flux rope. The corresponding "increase-to-decrease" change in the amount of toroidal magnetic flux agrees with the latest observational results. In a related numerical study, combined with observations, but focusing more on particular event studies, Vemareddy presents the topological analysis of two ARs that produced multiple CMEs, and shows the co-location between the remote-sensing signatures of flare ribbons, the brightening features as observed during flares, and the extrapolated magnetic field above the AR (indicative of magnetic flux rope structure at one particular point in time) based on a non-linear force-free field (NLFFF) model. This study confirms the role of magnetic flux rope in forming the core magnetic structures of CMEs. In another study, Xue et al. discuss the relatively rare spectroscopic observation of a prominence eruption leading to CME. A prominence is generally considered to be a proxy of magnetic flux rope. They are able to derive the change in plasma properties and kinematics associated with this prominence eruption.

As CMEs erupt and propagate into the interplanetary space, they may be detected in-situ when passing one or more spacecraft (thus identified and named as interplanetary CMEs, i.e., ICMEs), in addition to continuous remote-sensing observations such as coronagraphic imaging and radio waves. Such in-situ observations often yield a more complete and quantitative set of magnetic field and bulk 
plasma parameters, albeit only along one or more discrete points/ lines across the structures. This has enabled many studies of ICMEs (including the so-called magnetic clouds, or large-scale magnetic flux ropes) for decades. Wu et al. present a large study by using a simple model for magnetic flux ropes based on the Wind spacecraft in-situ measurements. They show modifications and improvement to the original model by applying to many Wind spacecraft events. Hu et al. present a detailed study of a magnetic flux rope event detected in-situ by two spacecraft that were approximately aligned radially but separated by about 0.2 astronomical units in radial distance. By applying both a twodimensional (2D) and a three-dimensional (3D) magnetic flux rope model, they reveal the reconstructed magnetic flux rope configurations characterized by spiral magnetic field lines. It is worth noting that the evolution of ICMEs in interplanetary space is also affected by the background solar wind medium. Therefore an improved characterization of the ambient solar wind conditions, e.g., via numerical means, is also being actively pursued, as demonstrated by Liu et al. They report a numerical study for improving the performance of a 3D MHD model of the interplanetary medium in which CMEs propagate. Shen et al. investigate the role of ICMEs in causing geomagnetic disturbances, especially by imposing strong and long-duration southward magnetic field onto near-Earth environment. They find that the interactions between large-scale structures including multiple ICMEs and interplanetary shock waves lead to intensified southward field component. A unique study by Zhang et al. examines the solar radio burst signatures often associated with the CMEs upon their eruptions and propagation. They employ the advanced deep learning algorithms to automatically identify and classify different types of radio bursts.

In addition to the direct employment of magnetic and plasma fluid property measurements of ICMEs, the discrete particle populations including streaming electrons and heavy ions are also important in probing the magnetic structures and other relevant conditions in the heliospheric environment. In addition, a series of studies looks into the relatively smallscale structures or those with more intermittent occurrence in the solar wind. Wang and Zhao find magnetic reconnection signatures that deviate from a quasi-steady state. Liu et al. perform a statistical study of small-scale magnetic flux ropes near the heliospheric current sheet (HCS) and find that they may mostly originate from the HCS. The role of magnetic reconnection in the interplanetary space on the change of topology/connectivity of magnetic flux ropes is further

\section{REFERENCES}

1. Temmer M. Space Weather: the Solar Perspective. Living Rev Solar Phys (2021) 18:4. doi:10.1007/s41116-021-00030-3

2. Feng HQ, Zhao GQ, Wang JM. Counterstreaming Electrons in Small Interplanetary Magnetic Flux Ropes. J Geophys Res Space Phys (2015) 120(10):175-10. doi:10.1002/2015JA021643

3. Jiang C, Wu ST, Feng X, Hu Q. Data-driven Magnetohydrodynamic Modelling of a Flux-Emerging Active Region Leading to Solar Eruption. Nat Commun (2016) 7:11522. doi:10.1038/ncomms11522 examined by Feng et al., using primarily the high-resolution magnetic field and plasma measurements, together with the suprathermal electrons (in the energy range of a few hundred $\mathrm{eV}$ ) that always stream away from the Sun along magnetic field lines. Different topologies can be inferred from these observations and the results indicate that magnetic reconnection is an active process for magnetic flux ropes in the interplanetary space. Ruan et al. study the solar wind dynamic pressure pulses (DPPs) often embedded within the ICME streams. DPPs are found to be related to intermittency, an intrinsic feature of solar wind turbulence. This type of studies helps improve our understanding of dynamic processes across multiple scales. Finally, Song et al. carry out a unique study by using the elemental composition measurements of heavy ions, including Helium, Carbon, Oxygen, and Iron ions with different charge states. By examining their variations along two spacecraft paths, widely separated but crossing the same ICME structure, it is concluded that significant inhomogeneity of composition exists along the axial dimension. Such analysis has significant implications for the origination of CMEs because the composition signatures are generally believed to preserve the conditions in solar corona where the formation of CMEs involving coronal plasmas takes place.

It is especially fitting and timely for this new collection to come to light, when we enter the era of Parker Solar Probe (PSP) [10], Solar Orbiter (SO) [11], and additional forthcoming spacecraft missions like Solar Ring (see Shen et al.), among others. We may conclude by quoting from $\mathrm{Hu}$ et al., "It is worth noting that as multi-spacecraft measurements become increasingly more available, .... new and exciting multi-messenger science will be enabled by using multiple analysis tools. It is highly anticipated that the constellations of current and future missions will usher in new frontiers in heliophysics research".

\section{AUTHOR CONTRIBUTIONS}

$\mathrm{QH}$ drafted the manuscript and all authors contributed to the final editing and proofreading of the manuscript.

\section{ACKNOWLEDGMENTS}

We thank all the authors, reviewers, the Chief Editor, and the Frontiers editorial staff, for making this collection a reality.

4. Hu Q. The Grad-Shafranov Reconstruction in Twenty Years: 1996 2016. Sci China Earth Sci (2017) 60:1466-94. doi:10.1007/s11430-0179067-2

5. Song H, Yao S. Characteristics and Applications of Interplanetary Coronal Mass Ejection Composition. Sci China Technol Sci (2020) 1-17.

6. Chen PF. Coronal Mass Ejections: Models and Their Observational Basis. Living Rev Solar Phys (2011) 8:1. doi:10.12942/lrsp-2011-1

7. Wyper PF, Antiochos SK, DeVore CR. A Universal Model for Solar Eruptions. Nature (2017) 544:452-5.

8. Amari T, Canou A, Aly JJ, Delyon F, Alauzet F. Magnetic Cage and Rope as the Key for Solar Eruptions, 554 (2018). p. 211-5. doi:10.1038/nature24671 
9. Jiang C, Feng X, Liu R, Yan X, Hu Q, Moore RL, et al. A Fundamental Mechanism of Solar Eruption Initiation. Nat Astron (2021). doi:10.1038/ s41550-021-01414-z

10. Fox N, Velli M, Bale S, Decker R, Driesman A, Howard R, et al. The Solar Probe Plus mission: Humanity's First Visit to Our star. Space Sci Rev (2016) 204:7-48.

11. Müller D, Cyr OC, Zouganelis I, Gilbert HR, Marsden R, et al. The Solar Orbiter mission - Science Overview. AઐA (2020) 642:A1. doi:10.1051/0004$6361 / 202038467$

Conflict of Interest: The authors declare that the research was conducted in the absence of any commercial or financial relationships that could be construed as a potential conflict of interest.
Publisher's Note: All claims expressed in this article are solely those of the authors and do not necessarily represent those of their affiliated organizations, or those of the publisher, the editors and the reviewers. Any product that may be evaluated in this article, or claim that may be made by its manufacturer, is not guaranteed or endorsed by the publisher.

Copyright $\odot 2021$ Feng, $\mathrm{Hu}$ and Song. This is an open-access article distributed under the terms of the Creative Commons Attribution License (CC BY). The use, distribution or reproduction in other forums is permitted, provided the original author(s) and the copyright owner(s) are credited and that the original publication in this journal is cited, in accordance with accepted academic practice. No use, distribution or reproduction is permitted which does not comply with these terms. 